\title{
The Natural History of Cervical Spondylotic Myelopathy and Ossification of the Posterior Longitudinal Ligament: A Review Article
}

\author{
Zaid Aljuboori $^{1}$, Maxwell Boakye ${ }^{1}$ \\ 1. Neurosurgery, University of Louisville School of Medicine, Louisville, USA \\ $\square$ Corresponding author: Zaid Aljuboori, zaid.aljuboori@yahoo.com \\ Disclosures can be found in Additional Information at the end of the article
}

\section{Abstract}

Cervical spondylotic myelopathy (CSM) is the chronic and slow deterioration of cervical spinal cord function. The pathophysiology of this condition is multifactorial, including compression, repetitive trauma, and vascular compromise of the spinal cord. Clinically, it presents as a progressive decline in patients' appendicular neurological function. The natural history of this disease varies but, it is well-known that the duration and degree of compression correlate negatively with prognosis. A mild degree of CSM tends to stabilize with potential improvement over time while more severe CSM tends to progress. Surgical intervention has shown to positively alter the natural history of the disease by halting the progression with some restoration of function.

Ossification of the posterior longitudinal ligament (OPLL) is a chronic disease that results in progressive ossification of the posterior longitudinal ligament of the spine. It commonly affects the cervical spine. The etiology is multifactorial in nature, including genetic and environmental factors. The progressive nature of this condition and the resultant cervical spinal stenosis make it one of the main causes of cervical myelopathy (CM). There is no medical therapy for this disease, and surgery is reserved for patients with CM caused by spinal cord compression. In this article, we review the different aspects of the natural history of both CSM and OPLL.

Received 04/09/2019

Review began 04/10/2019

Review ended 06/19/2019

Published 07/03/2019

๑) Copyright 2019

Aljuboori et al. This is an open access article distributed under the terms of the Creative Commons Attribution License CC-BY 3.0., which permits unrestricted use, distribution, and reproduction in any medium, provided the original author and source are credited.
Categories: Neurosurgery

Keywords: spine, myelopathy, opll, compressive, spondylosis

\section{Introduction And Background}

Cervical spondylotic myelopathy (CSM) is a clinical syndrome due to the disturbance of spinal cord function, both sensory and motor systems, due to a chronic degenerative process that results in progressive narrowing of the cervical spinal canal and resultant cord compression [13]. It usually presents as paresthesia, progressive loss of fine motor function, and imbalance. In advanced cases, patients do experience bowel and bladder dysfunction. The degenerative changes are intervertebral disc degeneration, osteophyte formation, and ligamentum flavum and facet hypertrophy [1-2,4]. The compression could be limited to a single level or may span multiple levels. It is the most common form of myelopathy in adults, especially in patients above 50 years of age $[2,4]$. The spinal canal narrowing predisposes the patient to chronic mechanical compression and vascular compromise of the spinal cord with additional repetitive trauma from the dynamic movement of the spine $[3,5]$ These insults result in degeneration of the spinal cord [5-6].

\section{How to cite this article}

Aljuboori Z, Boakye M (July 03, 2019) The Natural History of Cervical Spondylotic Myelopathy and Ossification of the Posterior Longitudinal Ligament: A Review Article. Cureus 11(7): e5074. DOI 10.7759 /cureus.5074 
Ossification of the posterior longitudinal ligament (OPLL) is a hyperostotic condition of the spine, where the posterior longitudinal ligament (PLL) becomes progressively calcified, often leading to symptomatic spinal canal stenosis [7-9]. Genetic, environmental, and biochemical factors have been implicated in the development of this disease with high prevalence in the Asian population [9-11]. OPLL also has familial distribution, it was radiographically evident in about a quarter of second-degree relatives, with a higher percentage in the siblings of OPLL patients [12]. It is considered a subtype of diffuse idiopathic skeletal hyperostosis (DISH) and can affect as many as half of the patients with DISH [10-11]. It is also prevalent among patients with cervical myelopathy [10]. There are four types of OPLL: 1) segmental, limited to the posterior surface of the vertebral bodies without crossing disc spaces; 2) continuous, spanning multiple levels with involvement of vertebral body and disc spaces; 3) mixed, a combination of segmental and continuous; and 4) other, limited to posterior disc spaces with some extension to the posterior vertebral body endplate [13-15].

\section{Review}

\section{Pathophysiology}

Cervical Spondylotic Myelopathy (CSM)

CSM is a multifactorial process involving static (stenosis) and dynamic (repetitive trauma to the cord during spine movement) factors [1]. Congenitally short pedicles, bulging intervertebral discs, and osteophyte formation are examples of static factors causing stenosis of the spinal canal [1]. Movement of the cervical spine leads to dynamic changes in the spinal cord; during extension, the cord becomes thicker and shorter, which exaggerates the existing compression, while flexion leads to the narrowing and draping of the spinal cord over osteophyte ridges. These dynamic changes are thought to cause CSM through increasing tension on the spinal cord [16]. In addition, Breig et al. suggested that vascular compromise due to mechanical compression of the arterioles supplying the spinal cord contributes to the development of CSM [16]. Cadaveric studies showed that the degree of compression correlates with the pathologic changes involving the gray matter (neuronal loss and necrosis) and white matter (demyelination and gliosis) of the spinal cord [5-6,17]. Ogino et al. published an autopsy report on nine patients with a history of CSM with an average duration of symptoms of 18.2 yrs. They showed a positive correlation between the anteroposterior (AP) compression ratio of the spinal cord, with histopathological changes of the cord substance. At AP compression ratios of $40 \%$ 44\%, $22 \%-39 \%$, and $12 \%-19 \%$, there was mild demyelination, gray matter cavitation with diffuse demyelination, extensive necrosis of gray matter, and gliosis of white matter, respectively [6].

These findings correlate with results from animal studies with experimental spinal stenosis and cord compression that demonstrated pathological changes mainly involving the gray matter with significant neuronal loss at the maximum area of compression [18].

\section{Ossification of the Posterior Longitudinal Ligament}

The pathophysiology of OPLL is poorly understood. Multiple studies have indicated an underlying genetic etiology [9-10,13,19-20]. The Investigation Committee on Ossification of the Spinal Ligaments in Japan performed a study on eight monozygotic and two dizygotic twins with OPLL. They reported that 75\% of monozygotic twins had OPLL, which indicated the involvement of genetic factors [15]. Nakajima et al. conducted a genetic study comparing patients with OPLL and healthy controls (1130 vs 7135 ) using genome-wide association. The authors found multiple genes associated with OPLL. These genes are Radial Spoke Head 9 Homolog (RSPH9), Serine/Threonine Kinase 38 like (STK38L), Hydroxyacid Oxidase 1 (HAO1A), Coiled-Coil Domain Containing 91 (CCDC91), and R-Spondin 2 (RSPO2). The RSPH9 and 
STK38L genes might be responsible for the membranous ossification of the PLL. Additionally, the HAO1A, RSPO2, and CCDC91 genes are responsible for the endochondral ossification [21]. A genome-wide linkage study by Karasugi et al. of 214 Japanese affected sib-pairs showed that several loci on chromosomes 1p, 2p, 7q, 16q, and 20p were associated with OPLL. Of note, sibpair is a linkage analysis of a dichotomous trait. It's a common method used to narrow search results for genes that influence complex diseases. Additionally, microsatellite markers mapping showed a significant association with locus D20S894 on Ch. 20p12 [22]. In addition, epidemiological studies have shown that adult-onset obesity and type 2 diabetes mellitus (DM) are independent risk factors for OPLL [23-24]. Elevated serum insulin has been associated with increased ossification in patients with OPLL. The exact mechanism is unclear, but it is thought that insulin might stimulate osteoprogenitor cells, which results in the ossification of the PLL [24-25]. Sleep duration and dietary habits have been associated with $n$ increased risk of OPLL as well [26-27]. Sleeping less than five or more than nine hours has been associated with increased risk of OPLL. High salt and low protein diet have been associated with OPLL as well but not cigarette smoking or alcohol consumption [26-27].

\section{Natural history}

Cervical Spondylotic Myelopathy

The natural history of CSM is described as a progressive worsening of signs and symptoms over time, but the rate and pattern of decline are unclear [1]. Two main patterns of CSM progression have been reported: (1) a slow worsening of function over time and (2) an extended period of stability of neurological function followed by expedited decline [28-29]. Clark et al., in their retrospective series of patients with CSM, observed that $75 \%$ of patients had a stable neurological function with episodic worsening while $20 \%$ had a slow progression over time [28]. Kadanka et al. evaluated the natural history of mild CSM modified Japanese Orthopedic Association score $(\mathrm{mJOA})>12$ treated either conservatively or surgically. The conservative group had 33 patients younger than 75 years with a one-year average duration of symptoms. At six months follow-up, 27\% remained stable while 72\% improved. At three years follow-up, $26 \%$ remained stable and $72 \%$ improved on the mJOA scale [30].

In addition to sensory and motor dysfunction, CSM has been shown to affect respiratory function. Bhagavatula et al., in their prospective study of pulmonary function test in 30 patients with CSM, showed that mean forced vital capacity (FVC) was 65\%, which was significantly lower than in the healthy control group $88 \%$. The mean forced expiratory volume in 1 second (FEV1) was 72\% in the CSM group and 96\% in the control group. The peak expiratory flow rate (PEFR) was $56.6 \%$ in the CSM group and 68.3\% in the control group. Additionally, there was a negative correlation between FVC and the Nurick score. [31] The exact mechanism for this subclinical respiratory dysfunction is not well-understood, but it was postulated that sensory, motor, and autonomic neural supply to the upper thorax through the C5-T1 nerve roots is the reason for this phenomenon [32].

Researchers have attempted to predict the development of CSM in patients with cervical stenosis using different strategies such as electrophysiological studies (EPS) and magnetic resonance spectroscopy (MRS). Kadanka et al., in their series of 30 patients with imagingconfirmed cervical spinal stenosis with no clinical evidence of CSM, performed a battery of electrophysiological studies including (motor-evoked potentials and sensory-evoked potentials). Fifty percent of patients had abnormal EPS; of this group, 30\% percent developed CSM during the two years follow-up while patients with normal EPS did not develop CSM [33].

MRS has been used to detect early cellular biochemical changes in the spinal cord in patients with advanced cervical spondylosis. Salomon et al. conducted a comparison study of patients with cervical spondylosis and healthy controls using regular magnetic resonance imaging (MRI) 
and MRS. They found that the relative concentration of choline to creatine (Cho/Cr) was significantly lower in healthy controls than in cervical stenosis patients with $\mathrm{T} 2$ cord signal change. There was no significant difference in $\mathrm{Cho/Cr}$ between healthy controls and patients with cervical stenosis, with no T2 cord signal change. They also observed that the choline to NAcetyl aspartate ratio (Cho/NAA) was significantly lower in the healthy controls in comparison to patients with cervical stenosis with or without T2 signal changes on MRI. They found a correlation between a lower Cho/NAA and higher mJOA score. They concluded that MRS can be used as a tool to detect early and late cellular biochemical changes of the spinal cord in patients with advanced cervical spondylosis. The choline/NAA ratio correlation with the mJOA score can provide a clinically useful radiographic biomarker to help manage patients with cervical spondylosis [34].

Roberts et al. conducted a retrospective study of 24 patients with a diagnosis of CSM based on clinical exam and myelography. They reported that the longevity and severity of symptoms correlated negatively with clinical improvement. They also reported that a symptom duration of more than 18 months was associated with a poor outcome [35]. Another study of 22 patients with a diagnosis of CSM by Sadasivan et al. showed that the longevity of symptoms prior to diagnosis affected outcomes. The patients in this study had symptoms for an average of 6.3 years before diagnosis; follow-up results showed that all patients deteriorated clinically [36].

Surgical decompression was proposed as a method to halt the progression of CSM, as well as to help patients by improving their neurological function and quality of life. Liu et al. conducted a study on 94 patients with CSM. They used a combination of the presence or absence of cord signal on MRI T2-weighted images along with $\mathrm{n}$ electromyography (EMG) analysis to predict functional outcome after surgical intervention. The EMG was either positive or negative. Positive results included lengthened duration (exceeding the normal value by $20 \%$ in the same age group), large-amplitude motor unit action potentials (MUAPs), and polyphasic motor units in at least two myotomes unilaterally or bilaterally. These electrophysiologic changes indicate anterior horn cell lesions. They stratified patients into four groups based on MRI and EMG results: $(-/-),(+/-),(-/+)$, and (+/+). Patients with no MRI cord signal and negative EMG had the best outcomes while patients with MRI cord signal and positive EMG had the worst outcome [37]. In addition, Hirai et al. performed a comparative study of anterior cervical decompression with fusion (ADF) and posterior decompression using laminoplasty. Seventynine patients received either ADF or laminoplasty with two years follow-up. ADF resulted in a higher mean JOA recovery rate at two years in comparison to laminoplasty. One potential limitation of this study is the average duration of symptoms before surgery was longer for the laminoplasty group (22.8 vs 39.8 months) [38]. Chagas et al. also evaluated the benefit of anterior decompression and fusion (ADF) in CSM patients; 39 patients with a mean duration of symptoms before surgery of 38.1 months were treated with a minimum follow-up of 18 months. Results showed that $64.1 \%$ of the patients had an improved Nurick scale postoperatively, $33.3 \%$ remained the same, and $2.6 \%$ got worse. They also found that patients younger than 60 years of age were more likely to benefit from surgery as compared to patients of age more than 60 years [39].

Manzano et al. compared expansile cervical laminoplasty (ECL) and cervical laminectomy and fusion (CLF) for multilevel cervical myelopathy. They enrolled 16 patients with a mean age of 59 years (range 41-75 years); nine patients randomized to the ECL group and seven patients to the CLF group. The duration of symptoms was 17 months for the ECL group and 20 months for the CLF group. Both groups were evaluated using the mJOA and Nurick myelopathy scales at baseline and after surgery. Postoperatively, both groups had an improvement in both the mJOA and Nurick scales, but only the Nurick scale improvement was statistically significant. In the ECL group, the mJOA score improved from $12.37+-1.2$ to 14.25 +- 0.96 while in the CLF group, the mJOA group improved from $12.57+-1.09$ to $13.57+-1.02$. They also reported that there were no significant differences in preoperative self-reported indexes between the two groups. 
Postoperatively, only the ECL group showed a statistically significant improvement in selfreported outcome measures, including neck, interscapular, and arm pain one year after surgery. The authors concluded that both procedures resulted in statistically significant improvements in neurological function. However, the ECL group had improvements in several self-reported outcome measures [40].

Fehlings et al. recently published clinical guidelines on the management of CSM. Their recommendations were as follow: (1) Surgical intervention is recommended for patients with moderate or severe CSM. (2) Patients with mild CSM can be offered surgical intervention or a trial of rehabilitation. Operative intervention should be considered if there is neurological deterioration or failure to improve with rehabilitation. (3) Prophylactic surgery should not be considered for patients with evidence of cervical cord compression but without myelopathy or signs and symptoms of radiculopathy. These patients should be counseled on the potential risks of progression and followed clinically. (4) Patients with evidence of cord compression and no myelopathy but have clinical evidence of radiculopathy are at a higher risk of developing myelopathy. These patients can be offered surgical intervention or a trial of rehabilitation with close follow-up. If the non-surgical option was pursued and the patients developed myelopathy, the patient should be managed according to the first or second recommendation depending on the severity of the clinical picture [41].

\section{Ossification of Posterior Longitudinal Ligament}

The natural history of OPLL has been well-studied, especially in the Asian population, researchers have evaluated radiographic progression, development of myelopathy, and the effects of different surgical techniques on the progression and clinical outcomes [13-14,42-43]. Katsumi et al. evaluated 41 OPLL patients with a mean age of $61.8 \mathrm{yrs}$, who were treated conservatively. The mean follow-up period was $25.6+-17.1$ months. Each patient was evaluated with a computed tomography (CT) scan twice, with a one-year interval to assess the progression of the disease. Computer software was used to analyze the patients' CT images and create a 3D model of the OPLL. The study demonstrated that there was no effect of gender, DM, family history of OPLL, and OPLL type and location on the rate of lesion progression.

Additionally, over the course of the follow-up period, the ossification volume increased by $7.5 \%$. Interestingly, they described that the annual rate of progression of OPLL differs by age group, with the highest rate afflicting the 30-49 years' age group [14].

Matsunaga et al. evaluated the radiographic progression of OPLL on both the axial and longitudinal planes over time. They enrolled 167 patients with OPLL, who were treated conservatively with a mean follow-up of 11.2 years. Forty-two percent of patients had axial progression; of these, $45 \%$ had more than a $2 \mathrm{~mm}$ increase in thickness while the rest had a less than $2 \mathrm{~mm}$ increase. Eighty-two percent of patients had an extension of the OPLL along the longitudinal plane [42]. In another study by Matsunaga et al. of the effects of strain distribution in the intervertebral disc space on the progression of OPLL, they enrolled 101 patients with OPLL of the cervical spine with no previous surgery. Dynamic lateral X-rays and a computer software program were used to analyze the strain distribution. They categorized the patients according to the type of OPLL: 23 continuous type, 25 mixed type, 49 segmental type, and 4 other types. Fifty-two (51\%) patients had progression over time. They found abnormal strain distribution in $62 \%$ (63) of the patients. Seventy-one percent (45) of this group showed the progression of ossification that matched the areas of abnormal strain distribution while only $18 \%$ of patients without abnormal strain distribution showed progression of OPLL [44].

Several studies have evaluated the development of cervical myelopathy in patients of OPLL. A multicenter cohort study was performed by the investigative committee on OPLL of the Japanese Ministry of Public Health, Labor, and Welfare to study the radiographic predictors for 
the development of myelopathy in patients with OPLL. They used X-rays, CT, and MRI. A total of 156 patients were enrolled with a mean follow-up of 10.3 years. The authors classified the ossification on the axial plane into the central and laterally deviated types. Thirty-nine patients had more than $60 \%$ compromise of the spinal canal by OPLL; all of these patients had myelopathy while only $49 \%$ of the patients with less than $60 \%$ canal compromise by OPLL had myelopathy. Additionally, the authors evaluated the dynamic movement of the cervical spine and found that it was higher in patients with myelopathy. They concluded that a lateral deviated axial ossification pattern and canal compromise of more than $60 \%$ are radiographic predictors for myelopathy [45].

Additionally, Matsunaga et al. studied the development of myelopathy in 359 patients with OPLL, with a minimum of 10 years follow-up (average 17.6 years). Ninety percent of patients had no myelopathy at the time of diagnosis. Upon follow up, 17\% developed myelopathy. Their analyses revealed that the myelopathy free rate was $71 \%$ at the 30 years follow-up in patients with OPLL and no myelopathy. Of the remaining 10\% of the population with OPLL and myelopathy at the time of diagnosis, the majority had a worsening of their myelopathy without treatment. In this cohort, all patients with canal compromise of more than $60 \%$ had myelopathy. They also found that range of motion (ROM) was higher in patients with myelopathy and less than $60 \%$ canal compromise. They concluded that prophylactic surgery in patients with OPLL without myelopathy may not be necessary. In addition, dynamic factors are of importance in the development of myelopathy, especially in patients with less than $60 \%$ canal compromise [43].

In regard to the effects of surgical intervention on the natural history of OPLL, Ogawa et al. retrospectively reviewed 72 patients with OPLL myelopathy who underwent open door laminoplasty with a minimum follow-up of five years. The mean preoperative JOA score was 9.2 +- 0.4. At the three years follow-up, the score improved to a mean of $14.2+-0.3$. This improvement was maintained at the five-year time point. They also reported postoperative progression of more than $2 \mathrm{~mm}$ of OPLL in $63.9 \%$ of the patients. In the axial plane OPLL thickness increased by a mean of $3.9 \mathrm{~mm}$, and in the longitudinal plane by a mean of $26.3 \mathrm{~mm}$. They reported late onset deterioration in 11 patients, which occurred more than 10 years after surgery [46]. Kawaguchi et al. described an association between laminoplasty done for OPLL myelopathy and the radiographic progression of OPLL. In their study, 73\% of patients had radiographic progression of OPLL after surgery. The longitudinal progression was worse than the axial progression. They also noticed that patients who had progression were younger (mean 53.3 years) than the non-progression group (mean 60.2 years). In addition, there was an association between the mixed or continuous type of ossification and the radiographic progression of OPLL [47]. Li et al. systematically reviewed studies that involved patients undergoing surgical decompression for OPLL myelopathy. They reported surgical complications, which included spinal cord injury, cerebrospinal fluid (CSF) leak, implant failure. CSF leak, hoarseness of voice, dyspnea, and dysphagia were more common with anterior approaches [48].

CT imaging has a valuable role in predicting the risk of a CSF leak in anterior approaches. It can be used to detect dural defects. The double-layer sign on CT describes the anterior and posterior rims of hyperdense regions (calcification) separated by a hypodense area (dural defect) due to OPLL growth into the dura [49].

Since the increased mobility caused by posterior surgical approaches resulted in a higher progression rate of OPLL in comparison to anterior approaches, an effort was made to minimize the complications associated with anterior approaches. The anterior floating method was described where a thin layer of OPLL was left adherent to the dura during corpectomy to avoid complications like a CSF leak. Matsuoka et al. studied the effects of anterior floating method decompression and fusion on 63 patients with cervical OPLL with a follow-up of more 
than 10 years. They reported that only three patients had a progression of OPLL (1-1.5 mm) at the operative site. Two of these three were attributed to inadequate thinning of the OPLL, and the third one was attributed to the side residual of OPLL. In 36 patients, there was a progression of OPLL in the segments adjacent to the operative site [50].

\section{Conclusions}

CSM is a chronic disease with different patterns of progression. It affects both the gray and white matter of the spinal cord, which leads to dysfunction of both motor and sensory systems. In addition, it affects the respiratory function of the patients. The length and severity of symptoms correlate negatively with prognosis. Surgical decompression may help halt disease progression and lead to variable improvement of function. OPLL is a multifactorial disease characterized by the chronic and progressive ossification of the PLL. Multiple genetic and biochemical aberrations have been linked to the development of this condition. Over time, it often leads to compressive myelopathy, which may require surgical intervention. Both the anterior and posterior surgical decompression approaches have been described, but the posterior approaches are more favored due to a lower complication rate.

\section{Additional Information}

\section{Disclosures}

Conflicts of interest: In compliance with the ICMJE uniform disclosure form, all authors declare the following: Payment/services info: All authors have declared that no financial support was received from any organization for the submitted work. Financial relationships: All authors have declared that they have no financial relationships at present or within the previous three years with any organizations that might have an interest in the submitted work. Other relationships: All authors have declared that there are no other relationships or activities that could appear to have influenced the submitted work.

\section{References}

1. Lebl DR, Hughes A, Cammisa FP Jr., O'Leary PF: Cervical spondylotic myelopathy: pathophysiology, clinical presentation, and treatment. HSS J. 2011, 7:170-178.

2. Bohlman HH, Emery SE: The pathophysiology of cervical spondylosis and myelopathy. Spine (Phila Pa 1976). 1988, 13:843-846.

3. Chatley A, Kumar R, Jain VK, Behari S, Sahu RN: Effect of spinal cord signal intensity changes on clinical outcome after surgery for cervical spondylotic myelopathy. J Neurosurg Spine. 2009, 11:562-567. 10.3171/2009.6.SPINE091

4. Sivaraman A, Bhadra AK, Altaf F, Singh A, Rai A, Casey AT, Crawford RJ: Skip laminectomy and laminoplasty for cervical spondylotic myelopathy: a prospective study of clinical and radiologic outcomes. J Spinal Disord Tech. 2010, 23:96-100. 10.1097/BSD.0b013e318198c92a

5. Ito T, Oyanagi K, Takahashi H, Takahashi HE, Ikuta F: Cervical spondylotic myelopathy. Clinicopathologic study on the progression pattern and thin myelinated fibers of the lesions of seven patients examined during complete autopsy. Spine (Phila Pa 1976). 1996, 21:827-833.

6. Ogino H, Tada K, Okada K, Yonenobu K, Yamamoto T, Ono K, Namiki H: Canal diameter, anteroposterior compression ratio, and spondylotic myelopathy of the cervical spine. Spine (Phila Pa. 1976). 1983, 8:1-15.

7. Fargen KM, Cox JB, Hoh DJ: Does ossification of the posterior longitudinal ligament progress after laminoplasty? Radiographic and clinical evidence of ossification of the posterior longitudinal ligament lesion growth and the risk factors for late neurologic deterioration. J Neurosurg Spine. 2012, 17:512-524. 10.3171/2012.9.SPINE12548

8. Iwasaki M, Okuda S, Miyauchi A, Sakaura H, Mukai Y, Yonenobu K, Yoshikawa H: Surgical strategy for cervical myelopathy due to ossification of the posterior longitudinal ligament: part 2: advantages of anterior decompression and fusion over laminoplasty. Spine (Phila Pa 1976). 2007, 32:654-660. 10.1097/01.brs.0000257566.91177.cb 
9. Inamasu J, Guiot BH, Sachs DC: Ossification of the posterior longitudinal ligament: an update on its biology, epidemiology, and natural history. Neurosurgery. 2006, 58:1027-1039. 10.1227/01.NEU.0000215867.87770.73

10. Matsunaga S, Yamaguchi M, Hayashi K, Sakou T: Genetic analysis of ossification of the posterior longitudinal ligament. Spine (Phila Pa. 1976). 1999, 24:937-938.

11. Sugrue PA, McClendon J Jr, Halpin RJ, Liu JC, Koski TR, Ganju A: Surgical management of cervical ossification of the posterior longitudinal ligament: natural history and the role of surgical decompression and stabilization. Neurosurg Focus. 2011, 30:3. 10.3171/2010.12.FOCUS10283

12. Terayama K: Genetic studies on ossification of the posterior longitudinal ligament of the spine. Spine (Phila Pa 1976). 1989, 14:1184-1191.

13. Matsunaga S, Sakou T: Epidemiology of ossification of the posterior longitudinal ligament . OPLL. K. Yonenobu et al. (ed): Springer-Verlag, Tokyo, Japan; 1997. 11-17. 10.1007/978-4431-67046-9_2

14. Katsumi K, Watanabe K, Izumi T, et al.: Natural history of the ossification of cervical posterior longitudinal ligament: a three dimensional analysis. Int Orthop. 2018, 42:835-842.

10.1007/s00264-017-3667-z

15. The ossification of the posterior longitudinal ligament of the spine (OPLL). The Investigation Committee on OPLL of the Japanese Ministry of Public Health and Welfare. Nihon Seikeigeka Gakkai Zasshi. 1981, 55:425-440.

16. Breig A, Turnbull I, Hassler O: Effects of mechanical stresses on the spinal cord in cervical spondylosis. A study on fresh cadaver material. J Neurosurg. 1966, 25:45-56.

10.3171/jns.1966.25.1.0045

17. Fujiwara K, Yonenobu K, Hiroshima K, Ebara S, Yamashita K, Ono K: Morphometry of the cervical spinal cord and its relation to pathology in cases with compression myelopathy. Spine (Phila Pa. 1976). 1988, 13:1212-1216. 10.1097/00007632-198811000-00002

18. al-Mefty O, Harkey HL, Marawi I, et al.: Experimental chronic compressive cervical myelopathy. J Neurosurg. 1993, 79:550-561. 10.3171/jns.1993.79.4.0550

19. Abiola R, Rubery P, Mesfin A: Ossification of the posterior longitudinal ligament: etiology, diagnosis, and outcomes of nonoperative and operative management. Global Spine J. 2016, 6:195-204. 10.1055/s-0035-1556580

20. Ikegawa S: Genomic study of ossification of the posterior longitudinal ligament of the spine . Proc Jpn Acad Ser B Phys Biol Sci. 2014, 90:405-412. 10.11005/jbm.2014.21.2.127

21. Nakajima M, Takahashi A, Tsuji T, et al.: A genome-wide association study identifies susceptibility loci for ossification of the posterior longitudinal ligament of the spine. Nat Genet. 2014, 46:1012-1016. 10.1038/ng.3045

22. Karasugi T, Nakajima M, Ikari K: Genetic Study Group of Investigation Committee on Ossification of the Spinal. J Bone Miner Metab. 2013, 31:136-143. 10.1007/s00774-012-0404-y

23. Akune T, Ogata N, Seichi A, Ohnishi I, Nakamura K, Kawaguchi H: Insulin secretory response is positively associated with the extent of ossification of the posterior longitudinal ligament of the spine. J Bone Joint Surg Am. 2001, 83:1537-1544. 10.2106/00004623-200110000-00013

24. Kobashi G, Washio M, Okamoto K, et al.: High body mass index after age 20 and diabetes mellitus are independent risk factors for ossification of the posterior longitudinal ligament of the spine in Japanese subjects: a case-control study in multiple hospitals. Spine (Phila Pa 1976). 2004, 29:1006-1010. 10.1097/00007632-200405010-00011

25. Shingyouchi Y, Nagahama A, Niida M: Ligamentous ossification of the cervical spine in the late middle-aged Japanese men. Its relation to body mass index and glucose metabolism. Spine (Phila Pa. 1976). 1996, 21:2474-2478.

26. Wang PN, Chen SS, Liu HC, Fuh JL, Kuo BI, Wang SJ: Ossification of the posterior longitudinal ligament of the spine. A case-control risk factor study. Spine (Phila Pa 1976). 1999, 24:142144.

27. Washio M, Kobashi G, Okamoto K, et al.: Sleeping habit and other life styles in the prime of life and risk for ossification of the posterior longitudinal ligament of the spine (OPLL): a casecontrol study in Japan. J Epidemiol. 2004, 14:168-173. 10.2188/jea.14.168

28. Clarke E, Robinson PK: Cervical myelopathy: a complication of cervical spondylosis . Brain. 1956, 79:483-510. 10.1093/brain/79.3.483

29. Matz PG, Anderson PA, Holly LT, et al.: The natural history of cervical spondylotic myelopathy. J Neurosurg Spine. 2009, 11:104-111. 10.3171/2009.1.SPINE08716 
30. Kadanka Z, Mares M, Bednanik J, et al.: Approaches to spondylotic cervical myelopathy: conservative versus surgical results in a 3-year follow-up study. Spine (Phila Pa 1976). 2002, 27:2205-2210.

31. Bhagavatula ID, Bhat DI, Sasidharan GM, Mishra RK, Maste PS, Vilanilam GC, Sathyaprabha TN: Subclinical respiratory dysfunction in chronic cervical cord compression: a pulmonary function test correlation. Neurosurg Focus. 2016, 40:3. 10.3171/2016.3.FOCUS1647

32. Toyoda H, Nakamura H, Konishi S, Terai H, Takaoka K: Does chronic cervical myelopathy affect respiratory function?. J Neurosurg Spine. 2004, 1:175-178. 10.3171/spi.2004.1.2.0175

33. Bednarik J, Kadanka Z, Vohanka S, Novotny O, Surelova D, Filipovicova D, Prokes B: The value of somatosensory and motor evoked potentials in pre-clinical spondylotic cervical cord compression. Eur Spine J. 1998, 7:493-500. 10.1007/s005860050113

34. Salamon N, Ellingson BM, Nagarajan R, Gebara N, Thomas A, Holly LT: Proton magnetic resonance spectroscopy of human cervical spondylosis at 3T. Spinal Cord. 2013, 51:558-563. 10.1038/sc.2013.31

35. Roberts AH: Myelopathy due to cervical spondylosis treated by collar immobilization . Neurology. 1966, 16:951-951. 10.1212/wnl.16.9.951

36. Sadasivan KK, Reddy RP, Albright JA: The natural history of cervical spondylotic myelopathy . Yale J Biol Med. 1993, 66:235-242.

37. Liu FJ, Sun YP, Shen Y, Ding WY, Wang LF: Prognostic value of magnetic resonance imaging combined with electromyography in the surgical management of cervical spondylotic myelopathy. Exp Ther Med. 2013, 5:1214-1218. 10.3892/etm.2013.934

38. Hirai T, Okawa A, Arai Y, et al.: Middle-term results of a prospective comparative study of anterior decompression with fusion and posterior decompression with laminoplasty for the treatment of cervical spondylotic myelopathy. Spine (Phila Pa 1976). 2011, 36:1940-1947. 10.1097/BRS.0b013e3181feeeb2

39. Chagas H, Domingues F, Aversa A, Vidal Fonseca AL, de Souza JM: Cervical spondylotic myelopathy: 10 years of prospective outcome analysis of anterior decompression and fusion. Surg Neurol. 2005, 31:35-36.

40. Manzano GR, Casella G, Wang MY, Vanni S, Levi AD: A prospective, randomized trial comparing expansile cervical laminoplasty and cervical laminectomy and fusion for multilevel cervical myelopathy. Neurosurgery. 2012, 70:264-277. 10.1227/NEU.0b013e3182305669

41. Fehlings MG, Tetreault LA, Riew KD, et al.: A clinical practice guideline for the management of patients with degenerative cervical myelopathy: recommendations for patients with mild, moderate, and severe disease and nonmyelopathic patients with evidence of cord compression. Global Spine J. 2017, 7:70-83. 10.1177/2192568217701914

42. Matsunaga S, Kukita M, Hayashi K, Shinkura R, Koriyama C, Sakou T, Komiya S: Pathogenesis of myelopathy in patients with ossification of the posterior longitudinal ligament. J Neurosurg. 2002, 96:168-172. 10.3171/spi.2002.96.2.0168

43. Matsunaga S, Sakou T, Taketomi E, Komiya S: Clinical course of patients with ossification of the posterior longitudinal ligament: a minimum 10-year cohort study. J Neurosurg. 2004, 100:245-248. 10.3171/spi.2004.100.3.0245

44. Matsunaga S, Sakou T, Taketomi E, Nakanisi K: Effects of strain distribution in the intervertebral discs on the progression of ossification of the posterior longitudinal ligaments. Spine (Phila Pa 1976). 1996, 21:184-189.

45. Matsunaga S, Nakamura K, Seichi A, et al.: Radiographic predictors for the development of myelopathy in patients with ossification of the posterior longitudinal ligament: a multicenter cohort study. Spine (Phila Pa 1976). 2008, 33:2648-2650. 10.1097/BRS.0b013e31817f988c

46. Ogawa Y, Toyama Y, Chiba K, et al.: Long-term results of expansive open-door laminoplasty for ossification of the posterior longitudinal ligament of the cervical spine. J Neurosurg Spine. 2004, 1:168-174. 10.3171/spi.2004.1.2.0168

47. Kawaguchi Y, Kanamori M, Ishihara H, Nakamura H, Sugimori K, Tsuji H, Kimura T: Progression of ossification of the posterior longitudinal ligament following en bloc cervical laminoplasty. J Bone Joint Surg Am. 2001, 83:1798-1802. 10.2106/00004623-200112000-00005

48. Li H, Dai LY: A systematic review of complications in cervical spine surgery for ossification of the posterior longitudinal ligament. Spine J. 2011, 11:1049-1057. 10.1016/j.spinee.2011.09.008

49. Yang H, Yang L, Chen D, Wang X, Lu X, Yuan W: Implications of different patterns of "double-layer sign" in cervical ossification of the posterior longitudinal ligament. Eur Spine J. 2015, 24:1631-1639. 10.1007/s00586-015-3914-1 


\section{Cureus}

50. Matsuoka T, Yamaura I, Kurosa Y, Nakai O, Shindo S, Shinomiya K: Long-term results of the anterior floating method for cervical myelopathy caused by ossification of the posterior longitudinal ligament. Spine (Phila Pa 1976). 2001, 26:241-248. 